\title{
Enhanced mechanical properties and anti-hydrothermal ageing behaviors of unsaturated polyester composites by carbon fibers interfaced with POSS
}

Dawei Jiang ${ }^{1,2}$, Lixin Xing ${ }^{1}$, Li Liu ${ }^{1, *}$, Shaofan Sun ${ }^{1}$, Qingbo Zhang ${ }^{1}$, Zijian Wu${ }^{1}$, Xingru Yan ${ }^{2}$, Jiang Guo, ${ }^{2}$ Yudong Huang ${ }^{1}$, Zhanhu Guo ${ }^{2, *}$

1. School of Chemical Engineering and Technology, Harbin Institute of Technology, Harbin, China

2. Integrated Composites Lab (ICL), Department of Chemical and Biomolecular Engineering, University of Tennessee, Knoxville, TN, 37996, USA

*Corresponding author. E-mail: liuli@hit.edu.cn; zguo10@utk.edu

\begin{abstract}
Trisilanollsobutyl polyhedral oligomeric silsesquioxane (SO 1450 POSS) has been uniformly grafted on the carbon fiber (CF) surface and the surface roughness (108.4 nm) of the CFs grafted with POSS was higher than that of the as-received CFs $(57.9 \mathrm{~nm})$. Dynamic contact angle analysis of the SO grafted CFs showed higher surface energy than that of the as-received CFs. After grafted with SO, the interlaminar shear strength of the unsaturated polyester (UPR) composites was $61 \mathrm{MPa}$, increased by $29.8 \%$ compared with that of the composites with the as-received CFs (47 MPa). The interfacial shear strength (IFSS) of SO grafted CFs/UPR composites was $88 \mathrm{MPa}$, significantly increased by $91 \%$ compared with that of the as-received CFs/UPR composites (46 MPa). The IFSS of the SO grafted CFs/UPR composites after hydrothermal ageing was $74 \mathrm{MPa}$ with a decrease of just $16 \%$ compared with that of the as-received $\mathrm{CFs} / \mathrm{UPR}$ composites (30 MPa) with a significant decrease of $35 \%$. The tensile strength of the SO grafted CFs/UPR composites (1280MPa) is $15 \%$ higher than that of the as-received CFs/UPR composites (1110 MPa). The impact energy of $1.52 \mathrm{~J}$ for the SO grafted $\mathrm{CFs} / \mathrm{UPR}$ composites is higher than $1.00 \mathrm{~J}$ for the as-received $\mathrm{CFs} / \mathrm{UPR}$ composites.
\end{abstract}

Keywords: A. Carbon fibres; A. Nano particles; B. Fibre/matrix bond; B. Interphase; B. Mechanical properties 


\section{Introduction}

The carbon fibers (CFs) reinforced polymer matrix composites have been used in many areas including aerospace, marine and automobile industries due to their excellent mechanical properties and low weight compared to traditional metal materials $[1,2]$. The CFs reinforced polymer matrix composites possess three parts including reinforcing CFs, hosting matrix and interphase between CFs and matrix. This interface between the fiber and the matrix makes a critical contribution to the performance of the composites and a good quality interface can result in good mechanical properties [3-5]. In recent years, many researchers focus on the modification of the interface between CFs and matrix using various methods such as physical coating treatment [6-8] and chemical grafting [9-15].

Unsaturated polyester resins (UPR) have been widely used due to their relatively low price, ease of handling and a good balance of mechanical and chemical properties, and have been a popular thermoset used as the polymer matrix in composites $[16,17]$. Usually, UPR reinforced with unmodified CFs have low interlaminar shear strength, interfacial strength and are subjected to stress cracking and brittle fracture due to the smooth and inert characteristics of the CFs [18-21]. As the applications of CFs/UPR composites become more and more important, CFs surface modification is required.

One useful method is to graft nanoparticles on CFs surface to increase the surface wettability, roughness or chemical bonding for an increased the interfacial strength between CFs and UPR [22].

Polyhedral oligomeric silesquioxanes (POSS) are organic-inorganic molecules, 
approximately 1 to $3 \mathrm{~nm}$ in diameter, with a general formula $\left(\mathrm{RSiO}_{1.5}\right)_{\mathrm{n}}$, where $\mathrm{R}$ is hydrogen or an organic group, such as alkyl, aryl or any of their derivatives [23]. Trisilanollsobutyl POSS (SO 1450) is a kind of POSS of its open-cage-like structure with an inorganic silsequioxane at the core, organic isobutyl groups attached at the corners of the cage and three active silanol functionalities. SO 1450 can be used for surface modification of fillers, such as metal oxides, and other materials. It is also effective as an additive to thermoplastics and thermoset polymers for improving the moisture resistance and process-ability $[24,25]$. SO 1450 possesses several distinct advantages (providing thermal and chemical stability, enhanced compatibility with the hosting polymer matrix or with the functional groups for chemically reacting with a polymer matrix) over traditional surface modifiers like silanes due to its hybrid molecule. For example, Yuan et al. grafted traditional silane coupling agent to carbon fiber surface to increase the mechanical properties of the carbon fibers reinforced phenolic resin, however at the sacrifice of decreasing the single fiber tensile strength. Zhu et al. grafted epoxy monomer through traditional silane coupling agent to increase the tensile strength of the carbon fibers reinforced epoxy resin.[26-28]. Our previous researches have demonstrated that the chemical bonding on the interface of CFs/UPR composites rather than roughness and wettability played the most important role in enhancing the interface strength to improve the mechanical properties. Though the effects of roughness and wettability played a weaker role than chemical bonding with a slightly improved interface strength by $6.2 \%[18,29]$, how to improve the mechanical properties significantly is not reported. Meanwhile, the anti-hydrothermal aging 
behaviors of the UPR composites were not studied yet.

In this paper, a proper interface between CFs and UPR with the combined action of roughness, wettability and chemical bonding was created by chemical grafting SO 1450 on the CFs surface. After the SO 1450 grafted on the CFs, the CFs roughness and wettability were improved with the formation of $\mathrm{Si}-\mathrm{O}-\mathrm{Si}$ and $\mathrm{Si}-\mathrm{O}-\mathrm{C}$ bonds at the interface of CFs/UPR composites. These bonds can increase some special properties like anti-hydrothermal ageing because destroying the $\mathrm{Si}-\mathrm{O}-\mathrm{Si}$ and $\mathrm{Si}-\mathrm{O}-\mathrm{C}$ bonds needs strong acid, strong base and more energy. The SO 1450 was introduced onto the CFs by directly reacting with the hydroxyl group of the CFs. It is easier to graft SO 1450 on CFs surface than other nanoparticles with different functional groups, which need graft intermediates on the CFs first and then graft the nanoparticles through the intermediate $[13,18,29-32]$. And the effects of the SO 1450 on the interface of CFs/UPR composites were investigated. The surface functional groups and surface chemical composition of the CFs were characterized by Fourier transform infrared spectroscopy (FTIR) and X-ray photoelectron spectroscopy (XPS), respectively. The surface morphologies of the CFs were observed by atomic force microscopy (AFM) and scanning electron microscope (SEM). The wettability and surface free energy of the untreated and functionalized fibers were investigated by dynamic contact angle analysis test (DCAT). The interfacial mechanical properties of the composites were characterized by short-beam bending test method (interlaminar shear strength, ILSS), single fiber pull-out test method (interfacial shear strength, IFSS), impact test method (impact toughness). The anti-hydrothermal ageing was characterized through IFSS. The tensile 
properties of the unidirectional CFs/UPR composites were tested in the fiber direction by using universal mechanical machine. The micro-mechanism of the composite interphase region was investigated by force modulation atomic force microscopy (FMAFM).

\section{Materials and experimental method}

\subsection{Materials}

AROPOL MR13006 unsaturated polyester resins (UPR) and low shrinkage agent LP4016 (reducing the volume shrinkage of UPR curing) were supplied by Ashland Inc., USA. Tert-butyl peroxybenzoate (TBPB, 98\% purity, ACROS ORGANICS Inc) was used as an initiator for UPR curing. $\mathrm{NaBH}_{4}$ (98\% purity) was purchased from Alfa Aesar. $\mathrm{H}_{2} \mathrm{SO}_{4}(98 \%), \mathrm{HNO}_{3}(68 \%)$ and tetrahydrofuran (THF) were purchased from Tianjin Dongli big chemical reagent factory. The un-sizing polyacrylonitrile (PAN) based carbon fibers (Chinese Carbon Fiber 300, CCF300) were obtained from WeiHai GuangWei Group $\left(12 \times 10^{3}\right.$ single filaments per tow, tensile strength of $3.26 \mathrm{GPa}$, average diameter of $7 \mu \mathrm{m}$, density of $1.76 \mathrm{~g} \mathrm{~cm}^{-3}$, Shandong, China). The trisilanollsobutyl POSS (SO 1450) was obtained from Hybrid Plastics Co., Inc. and were used as received. The chemical structures of POSS, TBPB, UPR and un-sizing CFs are shown in Fig. 1.

\subsection{Grafting procedures}

The CFs ( $4 \mathrm{~g}$ ) were oxidized in a 3:1 (v/v) mixture of concentrated $\mathrm{H}_{2} \mathrm{SO}_{4} / \mathrm{HNO}_{3}$ at $60{ }^{\circ} \mathrm{C}$ for $2 \mathrm{~h}$, washed with deionized water until water was neutral. The oxidized CFs were then reduced in a solution of $\mathrm{NaBH}_{4}(1.0 \mathrm{~g})$ in $200 \mathrm{~mL}$ anhydrous ethanol at room 
temperature for $20 \mathrm{~h}$. The mixture was heated to $78{ }^{\circ} \mathrm{C}$ and maintained for $4 \mathrm{~h}$. The products were washed with deionized water until the solution became neutral and then dried (the product is denoted as "CF-OH”). CF-OH was reacted with $\mathrm{SO} 140$ in $150 \mathrm{~mL}$ THF solution of $3.5 \mathrm{~g} \mathrm{SO} 1450$ at $65{ }^{\circ} \mathrm{C}$ for $12 \mathrm{~h}$ with mechanical stirring. The product was rinsed with excess THF to remove unreacted POSS, washed with deionized water until water became neutral, and then dried for other usages (the product is denoted as "CF-POSS"). The grafting of POSS on the surface of CFs is shown in Fig. 2.

\subsection{Fabrication of CFs/UPR composites}

The CFs reinforced UPR composites were prepared by compression molding method. The unidirectional prepreg carbon fibers were put into a mold to make composites. The detailed preparation description of the fiber prepreg is as follows. First, the fibers were wrapped around a metal frame with 11 circles. And then the resin solution of MR13006, LP4016 and TBPB used at a mixture ratio (weight) of 105:45:1 was made. The wrapped fibers were immerged into the resin solution for one hour and then the fibers were slightly rubbed till the resin was fully saturated into CFs. The prepreg fiber was completed. Briefly, the CFs/UPR composites were prepared by heating the samples at $80{ }^{\circ} \mathrm{C}$ for one hour without pressure, a pressure of $10 \mathrm{MPa}$ was applied at $100{ }^{\circ} \mathrm{C}$ for one hour, then the sample underwent $140{ }^{\circ} \mathrm{C}$ for one hour under $10 \mathrm{MPa}$, finally the mold was cooled down to room temperature with the pressure being maintained. The resin content of the composites was controlled at $30 \pm 1.5$ mass $\%$. The specimen dimensions were $20 \mathrm{~mm} \times 6.5 \mathrm{~mm} \times 2 \mathrm{~mm}$ for interlaminar shear strength, the specimen dimensions were $55 \mathrm{~mm} \times 6.5 \mathrm{~mm} \times 2 \mathrm{~mm}$ for impact test and the specimens dimensions 
were $250 \mathrm{~mm} \times 15 \mathrm{~mm} \times 1.0 \mathrm{~mm}$ for tensile strength test, respectively.

\subsection{Characterization}

The surface functional groups of the CFs were analyzed by Fourier Transform Infrared (FTIR) spectrophotometer (Nicolet, Nexus 670, USA) in Attenuated Total Reflectance (ATR) mode. Before the surface analysis, the CFs were dried for $2 \mathrm{~h}$ under vacuum at $150{ }^{\circ} \mathrm{C}$. In order to obtain a good optical contact, a very thin $\mathrm{KBr}$ layer was introduced between the prism and the CFs by using a KBr pelletizer. The FTIR spectra were acquired by scanning the specimens for 64 times in the wavenumber range of $400-4000 \mathrm{~cm}^{-1}$ with a resolution of $2 \mathrm{~cm}^{-1}$.

X-ray photoelectron spectroscopy (XPS, ESCALAB 220i-XL, VG, UK) was carried out to study the surface element of the CFs using a monochromated Al Ka source $(1486.6 \mathrm{eV})$ at a base pressure of $2 \times 10^{-9}$ mbar. The XPS was energy referenced to the C1s peak of graphite at $284.6 \mathrm{eV}$. The XPS Peak version 4.1 program was used for data analysis. The treatment effects on the fiber surface morphology were observed by using FEI Sirion 200 scanning electron microscope (SEM, Royal Dutch Philips Electronics Ltd., Netherlands) and atomic force microscopy (AFM, Solver-P47H, NT-MDT, Russia). Individual fiber was examined in Solver P47 AFM/STM system (NT-MDT Co.). AFM was also used to investigate the microstructures of the composites. The force modulation mode was adopted to study the cross-section surfaces of unidirectional $\mathrm{CFs} / \mathrm{UPR}$ composites and the relative stiffness of various phases, including the CFs, interface, and resin. The composites were first polished perpendicularly to the fiber axis using increasingly finer sand papers, and then with a $\mathrm{Cr}_{2} \mathrm{O}_{3}(50 \mathrm{~nm})$ suspension, finally 
washed with water under ultrasonication and dried for tests.

Dynamic contact angle and surface energy analyses between testing liquids (deionized water and diiodomethane, 99\% purity Alfa Aesar, USA) and single fiber were performed using a dynamic contact angle tensiometer (DCAT21, Data Physics Instruments, Germany).

The interlaminar shear strength (ILSS) of the CFs/UPR composites was measured on a universal testing machine (WD-1, Changchun, China) using a three point short beam bending test according to ASTM D2344. The specimens were conditioned and an enclosed testing space was maintained at room temperature. The measurements were at a cross-head speed of $2 \mathrm{~mm} / \mathrm{min}$.

The impact tests were carried out in a drop weight impact test system (9250HV, Instron, USA). The impact span was $40 \mathrm{~mm}$, the drop weight was $3 \mathrm{~kg}$ and the velocity was $2 \mathrm{~m} / \mathrm{s}$. Each reported data was an averaged value of 5 specimens.

A carbon fiber monofilament was fastened to a metal holder with adhesive tape. Some UPR droplets were applied to the monofilament with the embedded length of 60-80 $\mu \mathrm{m}$ using a fine-point applicator. The curing procedure was the same as that for the preparation of CFs/UPR composites. After curing, the interfacial shear strength (IFSS) was adopted to quantify the interfacial property between CFs and resin matrix by the interfacial evaluation equipment (Tohei Sangyo Co Ltd., Japan), at a crosshead displacement rate of $0.5 \mu \mathrm{m} \mathrm{s}^{-1}$.

The curing procedure was the same as that for the preparation of CFs/UPR composites. The IFSS was also measured after the samples boiled in $100{ }^{\circ} \mathrm{C}$ water at 
the time of $48 \mathrm{~h}$ for hydrothermal aging.

The tensile strength (TS) of a single filament was performed on an electronic mechanical universal material testing machine (Instron 5500R, USA) according to ASTM D 3379-75. A carbon fiber monofilament was adhered to a rectangular piece of paper with a rectangular hole in the middle of the paper. Before the test, the two long sides of the paper were cut. A gauge length of $20 \mathrm{~mm}$ and cross-head speed of 10 $\mathrm{mm} / \mathrm{min}$ were used for all the fiber samples. At least, 60 specimens were tested for each fiber type, and then the average value was considered as the tested value. The tensile strength of the unidirectional CFs/UPR composites was performed by using an Instron 5985 machine according to the ASTM D3039 with a cross-head speed of $2 \mathrm{~mm} / \mathrm{min}$ and a load cell of $250 \mathrm{kN}$. At least five specimens were tested for each type of composites. The obtained results are quoted as the average values with deviation from all tested specimens.

\section{Results and discussion}

\subsection{Surface chemical elemental composition}

Fig. 3(a\&b) shows the FTIR spectra of the as-received and SO 1450 grafted CFs. The as-received CFs are observed to have the bending vibration of adsorbed water and the $\mathrm{C}-\mathrm{H}$ stretching of methyl and methylene groups, few organic groups can be observed in the as-received fibers, Fig. 3(a). For the POSS grafted CFs, the broad band at about $880 \mathrm{~cm}^{-1}$ corresponds to the $\mathrm{Si}-\mathrm{C}$ stretching vibration, Fig. 3(b). The bands at around $1055 \mathrm{~cm}^{-1}$ correspond to the $\mathrm{Si}-\mathrm{O}-\mathrm{Si}$ or $\mathrm{Si}-\mathrm{OH}$ stretching vibrations. The band at $1102 \mathrm{~cm}^{-1}$ was assigned to the stretching vibration of $\mathrm{C}-\mathrm{O}$. The band at $1290 \mathrm{~cm}^{-1}$ corresponds to the asymmetric deformation vibration of the $\mathrm{CH}_{2}$ of $\mathrm{Si}-\mathrm{CH}_{2}$ due to the 
grafted POSS.

The surface composition of the as-received and grafted CFs was determined by XPS and the results are shown in Table 1 . The as-received CFs surface only contains carbon, oxygen and a small amount of nitrogen. After being grafted with SO 1450, the carbon content was decreased from $82.03 \%$ for the as-received CFs to $71.48 \%$ and the oxygen content was increased from $16.86 \%$ for the as-received CFs to $22.65 \%$ for the SO 1450 grafted CFs, and the silicon was observed with an element content of $4.74 \%$. The surface atomic $\mathrm{Si} / \mathrm{C}$ ratio was increased sharply from 0 to 0.07 for the $\mathrm{SO} 1450$ grafted CFs. The C1s XPS spectra of the as-received and SO 1450 grafted CFs are shown in Fig. 3(c\&d). The $\mathrm{C} 1 \mathrm{~s}$ spectrum of the as-received $\mathrm{CFs}$ shows $69.1 \% \mathrm{C}-\mathrm{C}, 21.6 \% \mathrm{C}-\mathrm{O}$, $5.9 \% \mathrm{C}=\mathrm{O}$ and $3.4 \% \mathrm{O}-\mathrm{C}=\mathrm{O}$. The $\mathrm{C} 1 \mathrm{~s}$ spectrum of the $\mathrm{SO} 1450$ grafted $\mathrm{CFs}$ shows $57.8 \% \mathrm{C}-\mathrm{C}, 35.9 \% \mathrm{C}-\mathrm{O}, 1.3 \% \mathrm{C}=\mathrm{O}, 3.7 \% \mathrm{O}-\mathrm{C}=\mathrm{O}$ and $1.3 \% \mathrm{C}-\mathrm{Si}$, respectively. Compared with the as-received $\mathrm{CFs}$, the $\mathrm{C}-\mathrm{O}$ was increased significantly from 21.6 to $35.9 \%$ for the SO 1450 grafted $\mathrm{CFs}$ and the $\mathrm{C}-\mathrm{Si}$ was observed due to the introduced SO 1450 on the CFs surface. Both FTIR and XPS analysis confirmed the successful grafting POSS on CFs surface.

\subsection{Surface topography and dynamic contact angle analysis of carbon fibers}

The SEM images of the as-received and SO 1450 grafted CFs are shown in Fig. 4(a

\&b), respectively. The surface of as-received CFs has many shallow grooves parallel distributed along the longitudinal direction of the CFs, Fig. 4(a). The SO 1450 particles are observed uniformly distributed on the CFs surface, Fig. 4(b). The more accurate microscopic and 3-dimensional topographies of CFs surface were performed by AFM. 
Fig. 4(c\&d) shows the as-received and SO 1450 grafted CFs. The carbon fibers surface roughness, calculated from the plane topography images by using the AFM software, is shown in Table 2. The as-received CFs have many relatively neat shallow grooves arising from the production process with a small roughness of $57.9 \mathrm{~nm}$. The CFs grafted with SO 1450 become much rougher $(108.4 \mathrm{~nm})$ due to the uniform POSS particles scattered on the surface and in the voids of the fiber grooves, Fig. 4(d).

The contact angle $\theta$ was determined from the mass change during the immersion of fibers in each test liquid, using Wilhelmy's Eq (1) [33]:

$$
\cos \theta=\frac{m g}{\pi \cdot d_{f} \cdot \gamma_{1}}
$$

where, $d_{f}$ is the fiber diameter, $g$ is the gravitational acceleration, and $\gamma_{1}$ is the surface tension of the test liquid. The surface energy $\left(\gamma_{f}\right)$, dispersion component $\left(\gamma_{f}^{d}\right)$ and polar component $\left(\gamma_{f}^{p}\right)$ of the CFs were estimated from the measured dynamic contact angles of the test liquids with known surface tension components and were calculated according to $\mathrm{Eq}(2 \& 3)$ :

$$
\gamma_{1}(1=\cos \theta)=2\left(\gamma_{1}^{p} \gamma_{f}^{p}\right)^{1 / 2}+2\left(\gamma_{1}^{d} \gamma_{f}^{d}\right)^{1 / 2} \quad \text { (2) } \quad \gamma_{f}=\gamma_{f}^{p}+\gamma_{f}^{d}
$$

where, $\gamma_{1}, \gamma_{1}^{d}$ and $\gamma_{1}^{p}$ are the liquid surface tension, its dispersion and polar component, respectively. Deionized water $\left(\gamma^{d}=21.8 \mathrm{~mJ} \cdot \mathrm{m}^{-1}, \gamma=72.8 \mathrm{~mJ} \cdot \mathrm{m}^{-1}\right)$ and diiodomethane $\left(\gamma^{d}=50.8 \mathrm{~mJ} \cdot \mathrm{m}^{-1}, \gamma=50.8 \mathrm{~mJ} \cdot \mathrm{m}^{-1}, 99 \%\right.$ purity, Alfa Aesar, USA) were used as the test liquids. Each measurement was repeated 5 times and the results were averaged. In this work, the surface wettability of the CFs was evaluated via a Cahn dynamic angle analysis system. The $\theta, \gamma, \gamma^{d}$ and $\gamma^{p}$ of the as-received and SO 1450 grafted CFs are summarized in Table 2. As shown in Table 2, after grafted with SO 
1450 , the $\theta$ was decreased to $30.2^{\circ}$ from $74.39^{\circ}$ for polar water, and was increased to $59.33^{\circ}$ from $56.31^{\circ}$ for non-polar diiodomethane. The $\gamma_{f}$ was obviously increased, and the $\gamma^{d}$ and $\gamma^{p}$ components of SO 1450 grafted CFs were 28.98 and $35.80 \mathrm{~mJ} \cdot \mathrm{m}^{-1}$, respectively. The $\gamma_{f}$ of the SO 1450 grafted CFs was calculated to be $64.76 \mathrm{~mJ} \cdot \mathrm{m}^{-1}$, significantly higher than that of the as-received CFs. The increased surface energy was due to the introduction of high polar functional group $(\mathrm{Si}-\mathrm{OH})$ on the CFs surface and the increased surface energy could effectively improve the wettability between the fibers and the resin.

\subsection{Interfacial property testing}

The force modulation AFM (FAFM) was used to characterize both surface topography and hardness diagram. The force modulation images of the interphase region between CFs and UPR matrix were shown in Fig. 4(e\&f). The clear interface region is observed after the SO 1450 grafted on the CFs, Fig. 4(e), however only two phases are observed for the as-received CFs reinforced UPR composites, Fig. 4(f). A uniform interface layer is formed due to the formation of $\mathrm{Si}-\mathrm{O}-\mathrm{Si}$ and $\mathrm{Si}-\mathrm{O}-\mathrm{C}$ bonds at the interface of CFs/UPR composites. There is a transition region (Fig.4f), at which the color is different from the matrix and the reinforcement, i.e., a little darker than matrix and a little lighter than reinforcement. This phenomenon can be seen clearly in Fig. $4 \mathrm{f}$ between CF and UPR. If there is an interphase, there would be a stiffness difference between matrix and reinforcement. The relative stiffness is indirectly indicated by the detection of magnitude (MAG, in units of $\mathrm{nA}$ ) of the flexural quantum of the mrcrocantilever. There are obviously different stiffness phases, i.e., CF, interphase and UPR, on the cross-section surface of the composites. The stiffness of interphase is higher than that of 
the UPR and lower than that of the CF.

Oxidation and grafting treatment will unavoidably introduce defects on the surface of fibers, which could decrease the fiber strength. However, compared with the strength of the as-received CFs (3.26 GPa), comparable fiber tensile strength is observed for the CFs grafted with SO 1450 (3.27 GPa). The strength of a single carbon fiber obeys the single Weibull distribution, i.e., the lower the Weibull shape parameter is, the more defects the fibers have. The tensile strength of a single fiber does not show discernable decrease although the defects were introduced after grafting. The results of the single fiber tensile test suggest that the chemical grafting of POSS on the fibers has no significant effects on the fiber tensile strength.

The interlaminar shear stress is important since it has a significant effect on the failure strength of the composite laminate. The interlaminar shear strength (ILSS, $\Gamma$ ) of the as-received and grafted CFs reinforced UPR composites has been characterized by short-beam bending tests to evaluate the interface strength of the composites. The $\Gamma$ for the short-beam test was calculated according to Eq. (4) [34].

$$
\Gamma=\frac{3 P_{b}}{4 b h}
$$

where $P_{b}$ is the maximum compression force at fracture in Newton, $b$ is the width of the specimen in $\mathrm{mm}$, and $h$ is the thickness in $\mathrm{mm}$. Each reported $\Gamma$ value was averaged for more than eight successful measurements. Fig. 5(a) shows the ILSS of the as-received, and SO 1450 grafted CFs reinforced UPR composites. The grafted fiber composites have higher ILSS (61 MPa) than that of the as-received fiber composites (47 MPa), an 
increase of $29.8 \%$ due to the combined effects of the enhanced mechanical interlocking, wettability and the introduced chemical bonding at the interface of the composites. The interfacial strength (IFSS) of CFs/UPR composites was calculated, Eq (5) [35],

$$
\text { IFSS }=\frac{E_{\min }}{\pi x d_{f} l_{e}}
$$

where $F_{\max }$ is the maximum load recorded, $d_{f}$ is the carbon fiber diameter, and $l_{e}$ is the embedded length of carbon fiber in the UPR. As shown in Fig. 5(b), the IFSS of SO 1450 grafted CFs is observed to have a higher value of $88 \mathrm{MPa}$, an increase of about $91 \%$ in comparison with that of the as-received CFs (46 MPa).

Fig. 5(c) shows the impact property testing results of the composites reinforced with the as-received and POSS grafted CFs. The initial, propagative, and total absorbed energy of the as-received CF composites were $0.21,0.79$ and $1.00 \mathrm{~J}$, respectively. After modification, the impact toughness is increased significantly for the composites containing SO 1450 grafted CFs. The initial, propagative, and total absorbed energy were $0.93,0.59$ and $1.52 \mathrm{~J}$ for the composites with CFs grafted with SO 1450, respectively. The results indicate a higher impact strength $(1.52 \mathrm{~J})$ than that without POSS grafted CFs $(1.00 \mathrm{~J})$. When the impact force is applied to the composites, the interface between CFs and UPR matrix serving as a shielding layer induces more cracks to prevent the crack tips to directly touch the CFs reinforcement that can efficiently absorb the fracture energy, resulting in an increased initial absorbed energy.

\subsection{Tensile properties of unidirectional CFs/UPR composites}

To further understand the combined effects of the enhanced mechanical interlocking, wettability and the introduced chemical bonding at the interface of the composites for 
the SO 1450 grafted CFs, the tensile strength of the unidirectional CFs/UPR composites was investigated by tensile test. Fig. 5(d) shows the tensile strength of the composites reinforced with the as-received and modified CFs. The tensile strength of the SO 1450 grafted CFs composites $(1280 \mathrm{MPa})$ is $15 \%$ higher than that of the as-received CFs composites (1110 MPa), Fig. 5(d). This increase is due to the introduction of SO 1450 nanoparticles on the CFs surface. The fiber surface nanostructures were altered by the SO 1450. The SO 1450 is the hybrid molecule with an inorganic silsequioane at the core, organic isobutyl groups attached at the corners of the cage and three active silanol functionalities. After the SO 1450 grafted on CFs surface, the roughness, surface energy, and chemical reaction of the CFs were significantly improved. These improvements enhanced the mechanical interlocking and chemical interlocking at the interface of the composites to give an enlarged tensile strength.

\subsection{Hydrothermal ageing of composites}

The hydrolysis and damage of the interface could decrease the mechanical properties of the composites. To better understand the role of SO 1450 in the enhancement of the interface in the composites, the hydrothermal ageing of the composites was investigated through the micro-composites boiling in the $100{ }^{\circ} \mathrm{C}$ for $48 \mathrm{~h}$. Fig. 5(b) shows the IFSS of the CFs/UPR composites after ageing. The as-received CFs/UPR composites after ageing have a IFSS value of $30 \mathrm{MPa}$, as compared with the as-received CFs/UPR composites before ageing (46 MPa), a significant decrease of $35 \%$. However, for the SO 1450 grafted CFs/UPR composites after ageing with a IFSS of $74 \mathrm{MPa}$, there is just $16 \%$ decrease. The Si-O-Si and Si-O-C bonds have been formed at the interface of the 
CFs/UPR composites after the SO 1450 grafted CFs, however, there is no bond formation between the as-received CFs and the resin. The damage of the Si-O-Si and Si-O-C bonds needs strong acid, strong base and more energy [36-38]. So better anti-hygrothermal ageing for the SO 1450 grafted CFs is due to the enhanced interface.

\subsection{Interface property of the impact fracture surfaces of composites}

Fig. 6(a\&b) shows the impact fracture surface of the composites. For the as-received CFs/UPR composites, Fig. 6(a), a lot of fibers were pulled out from the UPR matrix in a large area. The obviously observed interface de-bonding between CFs and UPR matrix and big holes in the matrix indicated a weak interfacial adhension. After grafted with SO 1450, few fibers were pulled out and the pull-out length of the fibers was very short due to the enhanced mechanical interlocking, wettability and the introduced chemical bonding at the interface of the composites, further confirming an improved interface between CFs and UPR matrix, consistent with the FMAFM, IFSS, ILSS and tensile test.

\section{Conclusion}

SO 1450 was uniformly grafted on the CFs surface and effectively enhanced the mechanical and anti-hydrothermal ageing properties of the CFs and UPR composites. The wettability and roughness of the CFs grafted with SO 1450 were improved compared with the as-received CFs. For the grafted CFs/UPR composites, the ILSS (61 $\mathrm{MPa})$, IFSS (88 $\mathrm{MPa})$, impact energy $(1.52 \mathrm{~J})$, tensile strength $(1280 \mathrm{MPa})$ and anti-hydrothermal ageing were dramatically increased compared with that of the as-received CFs/UPR composites (47, $46 \mathrm{MPa}, 1.43 \mathrm{~J}$ and $1110 \mathrm{MPa})$. Moreover, from the FMAFM and SEM observations of the impact fracture surfaces, a better interfacial 
adhesion between the CFs grafted with SO 1450 and the resin was observed. The improved interfacial strength for the SO 1450 grafted CFs/UPR composites was attributed to the combination of three factors: (1) the enhanced mechanical interlocking, (2) the improved wettability of the CFs and (3) the chemical bonding on the interface. The chemical bonding on the interface of $\mathrm{CFs} / \mathrm{UPR}$ composites can also improve the anti-hydrothermal ageing behaviors.

\section{Acknowledgement}

The authors would like to thank the Chang Jiang Scholars Program and National Natural Science Foundation of China (Nos.51073047 and 51173032) for financial support. Z. Guo acknowledges the National Science Foundation (NSF, CMMI 10-30755 and 13-14486) USA.

\section{References}

[1] Davis DC, Wilkerson JW, Zhu J, Hadjiev VG. A strategy for improving mechanical properties of a fiber reinforced epoxy composite using functionalized carbon nanotubes. Compos Sci Technol. 2011;71(8):1089-1097.

[2] Zhang X, Fan X, Yan C, Li H, Zhu Y, Li X, et al. Interfacial microstructure and properties of carbon fiber composites modified with graphene oxide. ACS Appl Mater Inter. 2012;4(3):1543-1552.

[3] Choi M, Jeon B, Chung I. The effect of coupling agent on electrical and mechanical properties of carbon fiber/phenolic resin composites. Polymer. 2000;41(9):3243-3252.

[4] Delamar M, Desarmot G, Fagebaume O, Hitmi R, Pinsonc J, Savéant J-M. Modification of carbon fiber surfaces by electrochemical reduction of aryl diazonium salts: Application to carbon epoxy composites. Carbon. 1997;35(6):801-807.

[5] Ehlert GJ, Sodano HA. Zinc oxide nanowire interphase for enhanced interfacial strength in lightweight polymer fiber composites. ACS Appl Mater Inter. 2009;1(8):1827-1833.

[6] Sager R, Klein P, Lagoudas D, Zhang Q, Liu J, Dai L, et al. Effect of carbon nanotubes on the interfacial shear strength of T650 carbon fiber in an epoxy matrix. Compos Sci Technol. 2009;69(7):898-904.

[7] Zhang X, Song Y, Huang Y. Properties of silsesquioxane coating modified carbon fibre/polyarylacetylene composites. Compos Sci Technol. 2007;67(14):3014-3022.

[8] Guo H, Huang Y, Liu L, Shi X. Effect of epoxy coatings on carbon fibers during 
manufacture of carbon fiber reinforced resin matrix composites. Mater Des.. 2010;31(3):1186-1190.

[9] Ehlert GJ, Galan U, Sodano HA. Role of surface chemistry in adhesion between $\mathrm{ZnO}$ nanowires and carbon fibers in hybrid composites. ACS Appl Mater Inter. 2013;5(3):635-645.

[10] Zhao F, Huang Y, Liu L, Bai Y, Xu L. Formation of a carbon fiber/polyhedral oligomeric silsesquioxane/carbon nanotube hybrid reinforcement and its effect on the interfacial properties of carbon fiber/epoxy composites. Carbon. 2011;49(8):2624-2632.

[11] Zhu J, Wei S, Ryu J, Budhathoki M, Liang G, Guo Z. In situ stabilized carbon nanofiber (CNF) reinforced epoxy nanocomposites. $J$ Mater Chem. 2010;20(23):4937-4948.

[12] Le Bonheur V, Stupp SI. Grafting of a functionalized side-chain liquid-crystal polymer on carbon fiber surfaces: novel coupling agents for fiber/polymer matrix composites. Chem Mater. 1993;5(9):1287-1292.

[13] He X, Zhang F, Wang R, Liu W. Preparation of a carbon nanotube/carbon fiber multi-scale reinforcement by grafting multi-walled carbon nanotubes onto the fibers. Carbon. 2007;45(13):2559-2563.

[14] Peng Q, Li Y, He X, Lv H, Hu P, Shang Y, et al. Interfacial enhancement of carbon fiber composites by poly(amido amine) functionalization. Compos Sci Technol. 2013;74:37-42.

[15] Li Y, Li Y, Ding Y, Peng Q, Wang C, Wang R, et al. Tuning the interfacial property of hierarchical composites by changing the grafting density of carbon nanotube using 1,3-propodiamine. Compos Sci Technol. 2013;85:36-42.

[16] Aziz SH, Ansell MP, Clarke SJ, Panteny SR. Modified polyester resins for natural fibre composites. Compos Sci Technol. 2005;65(3-4):525-535.

[17] Mishra S, Mohanty AK, Drzal LT, Misra M, Parija S, Nayak SK, et al. Studies on mechanical performance of biofibre/glass reinforced polyester hybrid composites. Compos Sci Technol. 2003;63(10):1377-1385.

[18] Jiang D, Liu L, Long J, Huang Y, Wu Z, Yan X, et al. Reinforced Unsaturated Polyester Composites by Chemically Grafting Amino-POSS onto Carbon Fibers with Active Double Spiral Structural Spiralphosphodicholor. Compos Sci Technol. 2014.

[19] Jiang D, Liu L, Zhao F, Zhang Q, Sun S, He J, et al. Improved interfacial properties of carbon fiber/unsaturated polyester composites through coating polyhedral oligomeric silsesquioxane on carbon fiber surface. Fibers Polym. 2014;15(3):566-573.

[20] Peng Q, Li Y, He X, Lv H, Hu P, Shang Y, et al. Interfacial enhancement of carbon fiber composites by poly(amido amine) functionalization. Compos Sci Technol. 2013;74:37-42.

[21] Li Y, Li Y, Ding Y, Peng Q, Wang C, Wang R, et al. Tuning the interfacial property of hierarchical composites by changing the grafting density of carbon nanotube using 1,3-propodiamine. Compos Sci Technol. 2013;85:36-42.

[22] Zhang X, Alloul O, He Q, Zhu J, Verde MJ, Li Y, et al. Strengthened magnetic epoxy nanocomposites with protruding nanoparticles on the graphene nanosheets. Polymer. 2013;54(14):3594-3604.

[23] Monticelli O, Fina A, Cozza ES, Prato M, Bruzzo V. POSS vapor phase grafting: a 
novel method to modify polymer films. J Mater Chem. 2011;21(44):18049-18054.

[24] Deng J, Polidan JT, Hottle JR, Farmer-Creely CE, Viers BD, Esker AR. Polyhedral oligomeric silsesquioxanes: a new class of amphiphiles at the air/water interface. J Am Chem Soc. 2002;124(51):15194-15195.

[25] Godnjavec J, Znoj B, Veronovski N, Venturini P. Polyhedral oligomeric silsesquioxanes as titanium dioxide surface modifiers for transparent acrylic UV blocking hybrid coating. Progress in Organic Coatings. 2012;74(4):654-659.

[26] Zhu J, Wei S, Ryu J, Budhathoki M, Liang G, Guo Z. In situ stabilized carbon nanofiber (CNF) reinforced epoxy nanocomposites. J Mater Chem. 2010;20(23):4937.

[27] Zhu J, Wei S, Yadav A, Guo Z. Rheological behaviors and electrical conductivity of epoxy resin nanocomposites suspended with in-situ stabilized carbon nanofibers. Polymer. 2010;51(12):2643-2651.

[28] Yuan H, Wang C, Zhang S, Lin X. Effect of surface modification on carbon fiber and its reinforced phenolic matrix composite. Appl Surf Sci. 2012;259:288-293.

[29] Guo Z, Ding D, Yan X, Zhang X, Huang Y-D, Wei S, et al. Interfacially Reinforced Unsaturated Polyester Composites by Chemically Grafting Different Functional POSS onto Carbon Fibers. J Mater Chem A. 2014.

[30] Zhao F, Huang Y. Grafting of polyhedral oligomeric silsesquioxanes on a carbon fiber surface: novel coupling agents for fiber/polymer matrix composites. J Mater Chem. 2011;21(11):3695-3703.

[31] Zhao F, Huang Y. Preparation and properties of polyhedral oligomeric silsesquioxane and carbon nanotube grafted carbon fiber hierarchical reinforcing structure. J Mater Chem. 2011;21(9):2867-2870.

[32] Peng Q, He X, Li Y, Wang C, Wang R, Hu P, et al. Chemically and uniformly grafting carbon nanotubes onto carbon fibers by poly (amidoamine) for enhancing interfacial strength in carbon fiber composites. J Mater Chem. 2012;22(13):5928-5931.

[33] Qian H, Bismarck A, Greenhalgh ES, Kalinka G, Shaffer MS. Hierarchical composites reinforced with carbon nanotube grafted fibers: the potential assessed at the single fiber level. Chem Mater. 2008;20(5):1862-1869.

[34] Li M, Matsuyama R, Sakai M. Interlaminar shear strength of C/C-composites: the dependence on test methods. Carbon. 1999;37(11):1749-1757.

[35] Zhandarov S, Mäder E. Characterization of fiber/matrix interface strength: applicability of different tests, approaches and parameters. Compos Sci Technol. 2005;65(1):149-160.

[36] Liu YR, Huang YD, Liu L. Effects of TriSilanolIsobutyl-POSS on thermal stability of methylsilicone resin. Polym Degrad Stab. 2006;91(11):2731-2738.

[37] Ciolacu FCL, Choudhury NR, Dutta N, Kosior E. Molecular level stabilization of poly (ethylene terephthalate) with nanostructured open cage trisilanolisobutyl-POSS. Macromolecules. 2007;40(2):265-272.

[38] Gnanasekaran D, Madhavan K, Reddy B. Developments of polyhedral oligomeric silsesquioxanes (POSS), POSS nanocomposites and their applications: a review. J Sci Ind Res. 2009;68(6):437-464. 
Table 1. Surface chemical composition of CFs with and without POSS grafted.

\begin{tabular}{clccccl}
\hline & \multicolumn{6}{c}{ Element content (at\%) } \\
\cline { 2 - 7 } Carbon fibers & $\mathrm{C}$ & $\mathrm{O}$ & $\mathrm{N}$ & $\mathrm{Si}$ & $\mathrm{O} / \mathrm{C}$ & $\mathrm{Si} / \mathrm{C}$ \\
\hline As-received CFs & 82.03 & 16.86 & 1.1 & - & 0.20 & - \\
CF-trisilanollsobutyl POSS & 71.48 & 22.65 & 1.13 & 4.74 & 0.32 & 0.07 \\
\hline
\end{tabular}

Table 2. Contact angle, surface energy and roughness of CFs with and without POSS.

\begin{tabular}{|c|c|c|c|c|c|c|}
\hline \multirow{2}{*}{$\mathrm{CF}$} & \multicolumn{2}{|c|}{ Contact angle $\left({ }^{\circ}\right)$} & \multicolumn{3}{|c|}{ Surface energy $\left(\mathrm{mJ} \cdot \mathrm{m}^{-1}\right)$} & \multirow{2}{*}{$\mathrm{Ra}(\mathrm{nm})$} \\
\hline & Water & Diiodomethane & $\gamma^{\mathrm{d}}$ & $\gamma^{p}$ & $\gamma$ & \\
\hline As-received CFs & 74.39 & 56.31 & 23.82 & 10.64 & 34.46 & $57.9 \pm 1.1$ \\
\hline CF-trisilanollsobutyl POSS & 30.20 & 59.33 & 28.96 & 35.80 & 64.76 & $108.4 \pm 4.0$ \\
\hline
\end{tabular}

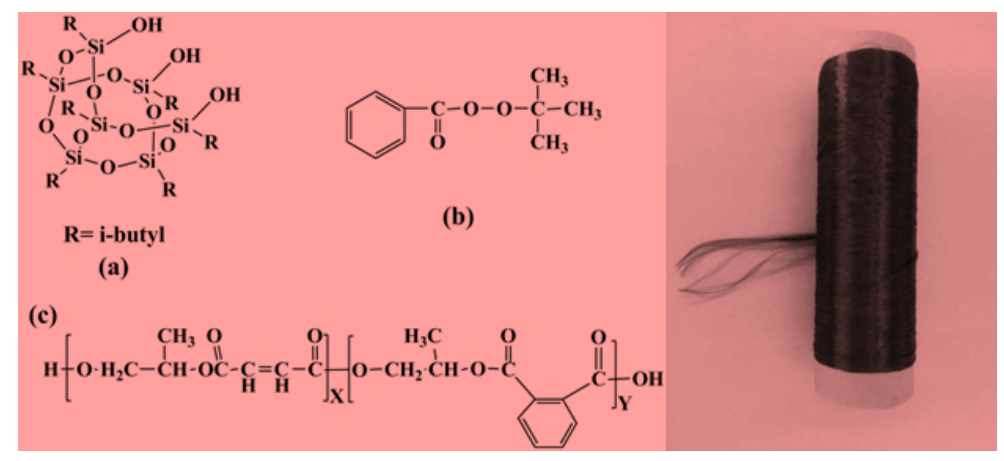

Fig. 1. Chemical structure of (a) trisilanollsobutyl POSS, (b) TBPB, (c) UPR, and image of un-sizing CFs.

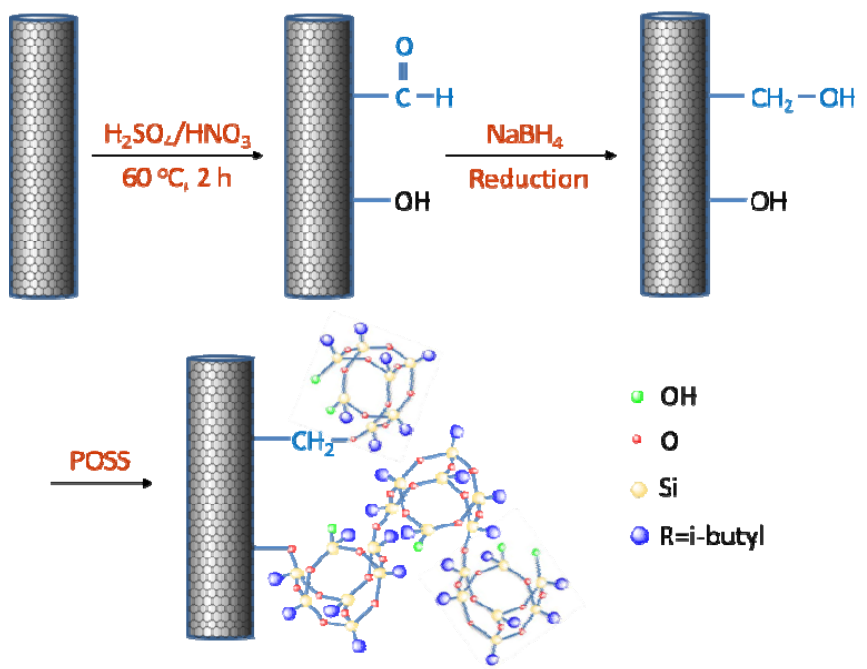

Fig. 2. Schematic illustration of the grafting procedures, not to scale. 

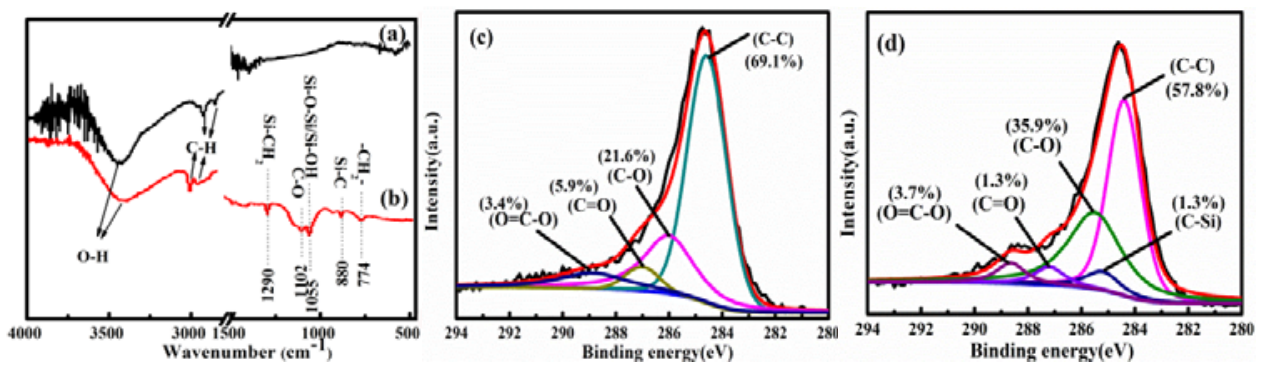

Fig. 3. FTIR-ATR (a), as-received (b) SO 1450 grafted CFs, and C1s spectra of XPS (c) as-received and (d) SO 1450 grafted CFs.
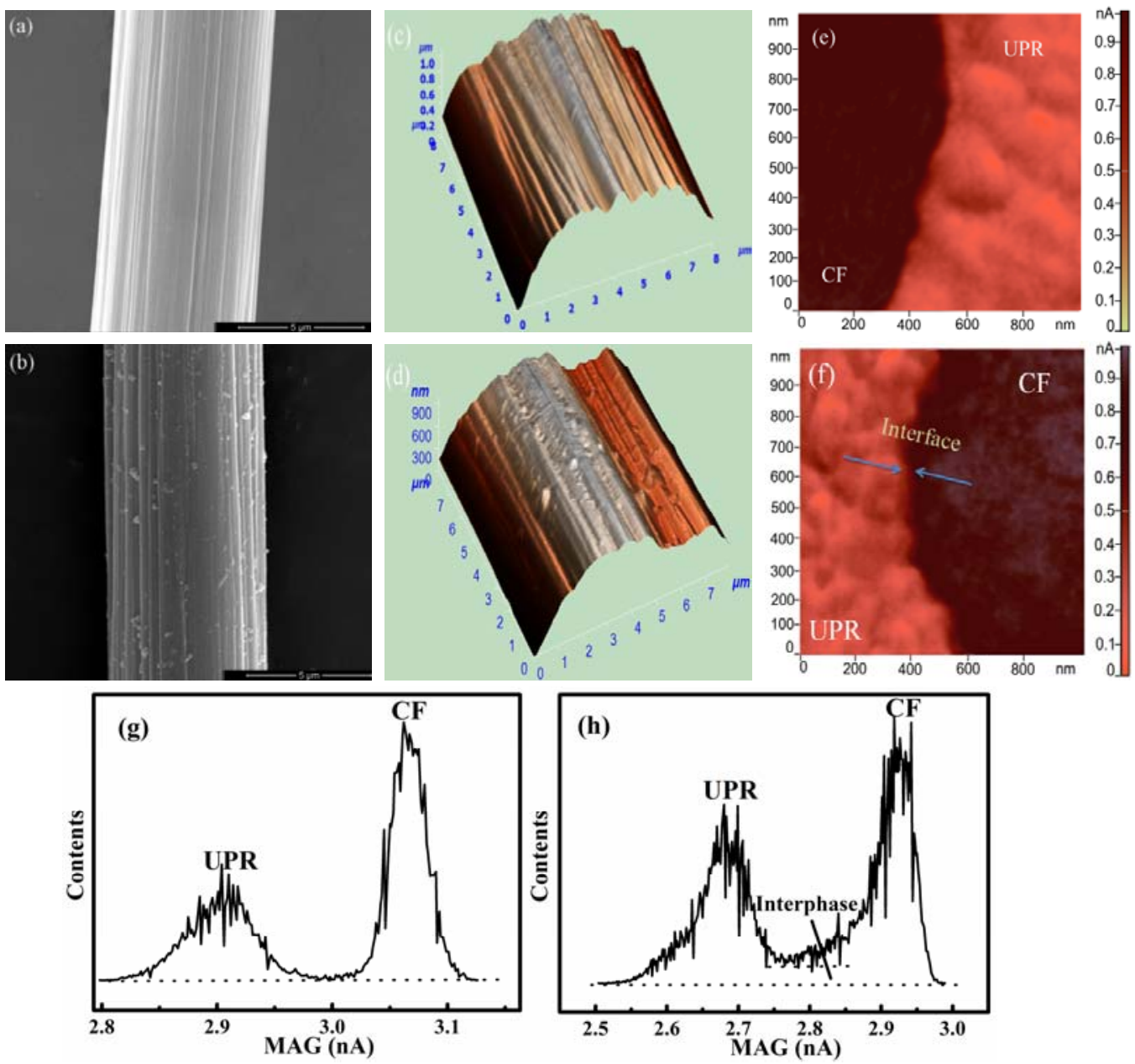

Fig. 4. SEM, AFM three-dimensional and force modulation AFM images of (a),(c),(e) as-received, and (b),(d),(f) SO 1450 grafted CFs, cross-sectional probability histogram of the interphase images of (g) as-received and (h) SO 1450 grafted CFs. 

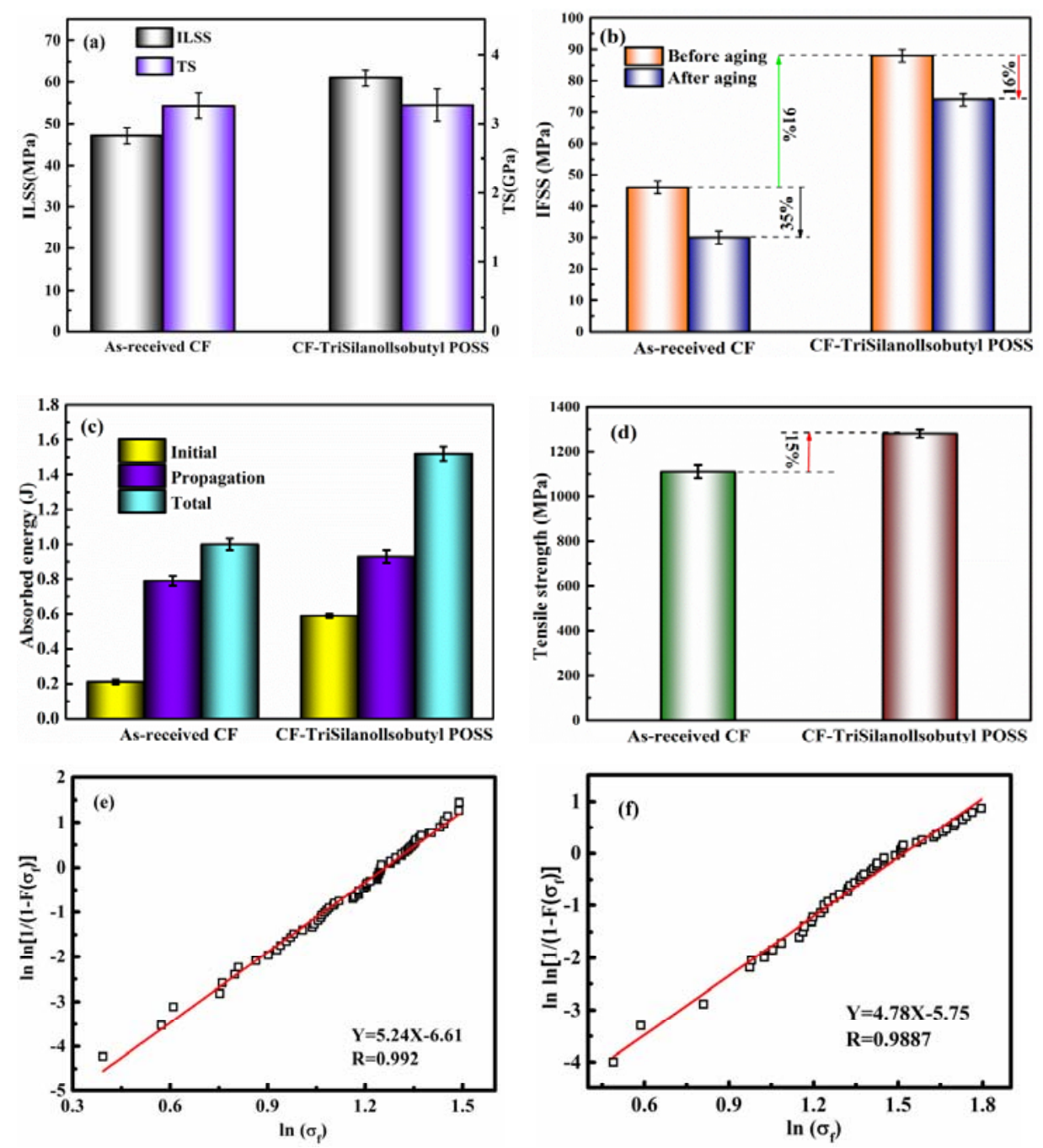

Fig. 5. (a) Tensile strength of single fiber and ILSS, (b) IFSS before and after hydrothermal aging, (c) impact test, (d) tensile strength of composites reinforced with the as-received and modified CFs, and (e), (f) Weibull probability plot of single fiber tensile strength of the as-received and modified CFs.
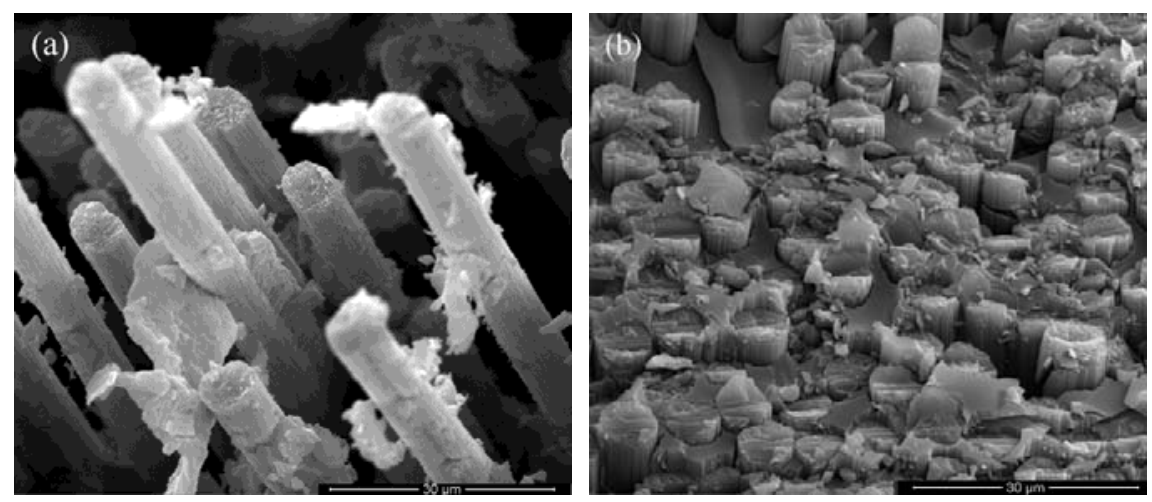

Fig. 6. Morphologies of the fractured surface of $\mathrm{CFs} / \mathrm{UPR}$ composites with (a) as-received, and (b) SO 1450 grafted CFs. 